\title{
“Cuanto más practiques, mejor lo harás”: evaluación multi-informante en el contexto de un curso e-learning
}

Alexandra Morales \& Mireia Orgilés

Universidad Miguel Hernández de Elche, España

\begin{abstract}
The objective of this work was to describe the process of multi-informant evaluation of an activity fot the resolution of clinical cases in an e-learning course with Edx platform. Possible differences in anxiety, self-efficacy and task performance were analyzed among the three informants: the students, their classmates and the professor. The students elaborated educational videos in which they solved a clinical case following the instructions of the professor. The evaluations were conducted independently with Google Forms. The results showed differences in the variables evaluated among the evaluations of the three informants. The peer and professor evaluations were more similar to each other, and differed from the students' self-assessments. Although the scores were acceptable, the students tended to self-evaluate more anxious and more negatively in their performance compared to the evaluations of the peers and the professor. Older students self-evaluated more negatively in terms of performance and self-efficacy than their younger peers. The multi-informant evaluation is applicable to other educational contexts to provide a more enriching vision of the learning process. We reflect on this pilot evaluation experience and propose some ideas for the future.
\end{abstract}

Keywords: evaluation, multi-informant, EdX, competencies, clinical cases.

\section{Resumen}

El objetivo de este trabajo fue describir el proceso de evaluación multiinformante de una actividad docente de resolución de casos clínicos en un curso e-learning con la plataforma Edx. Se analizan posibles diferencias en ansiedad, autoeficacia y desempeño de la tarea entre los tres informantes: los propios alumnos, sus compañeros y el profesor. Los alumnos elaboraron vídeos docentes en los que resolvían un caso clínico siguiendo las instrucciones del profesor. Las evaluaciones se realizaron independientemente con Google Forms. Los resultados arrojaron diferencias entre las evaluaciones de los tres informantes en las variables evaluadas. Las evaluaciones de los compañeros y de los profesores fueron más similares entre sí, y se diferenciaron de las autoevaluaciones de los alumnos. Aunque las calificaciones fueron notables, los alumnos tendieron a autoevaluarse más ansiosos y más negativamente en su ejecución respecto a las valoraciones de los compañeros y el profesor. Los alumnos de mayor edad se autoevaluaron más negativamente en términos de desempeño y autoeficacia que los compañeros de menor edad. La evaluación multi-informante es 
aplicable a otros contextos educativos para aportar un visión más enriquecedora del proceso de aprendizaje. Se reflexiona sobre esta experiencia piloto de evaluación y se proponen algunas ideas para el futuro.

Palabras clave: evaluación, multi-informante, EdX, competencias, casos clínicos.

\section{Introducción}

Plataformas docentes como Edx ofrecen un entorno de aprendizaje dinámico y atractivo para el alumno, alternativo o complementario a las clases presenciales. Son múltiples las variantes que ofrecen los entornos virtuales de aprendizaje. En este sentido, los recursos y objetivos del curso/asignatura son determinantes para seleccionar el formato y la metodología de aplicación y evaluación. Habitualmente estos cursos constan de videconferencias, clases prácticas, debates y foros de intercambio de opiniones. Además, ponen a disposición del equipo docente diversos sistemas de evaluación para verificar los objetivos de aprendizaje alcanzados durante el curso.

En los últimos años las universidades y otros centros docentes se han esforzado por incorporar los entornos virtuales a su oferta docente, con el fin de facilitar la formación de un público cada vez más diverso e internacional. Entre las modalidades disponibles están los cursos e-learning, que se caracterizan por presentar contenidos de formación cerrados, requieren de matricula previa para acceder y acreditan los aprendizajes a través de la evaluación de los alumnos (UAB, 2017). Actualmente se ofrece un importante número de cursos y másteres oficiales en formato e-learning, con la ventaja principal de facilitar el aprendizaje a quienes no pueden asistir presencialmente por diversas razones (Hernández y Carril, 2012). Sin embargo, una de las principales limitaciones de los entornos virtuales de aprendizaje es la dificultad para evaluar los contenidos prácticos a distancia, de forma controlada y bajo la supervisión del profesor.

La metodología de evaluación en el proceso de aprendizaje puede determinar los resultados académicos (p.ej., examen tipo test, una prueba situacional o un examen oral). Por parte del alumno, dos de los factores que pueden influir en su desempeño y en la transferencia de los contenidos teóricos a la práctica son: el nivel de ansiedad experimentado durante la actividad y su percepción de eficacia para poner en práctica las habilidades necesarias y para alcanzar los objetivos docentes establecidos (Álvarez y Muñoz, 2018; Contreras et al., 2005).

La ansiedad ha sido definida como una respuesta adaptativa al medio que nos permite anticipar situaciones díficiles y movilizar los recursos necesarios para afrontarlas (Orgilés, 2014; Spielberger, 1979). Hay evidencia de que un nivel moderado de ansiedad favorece el rendimiento del estudiante (Sue, 1996). No obstante, cuando la ansiedad es alta o desproporcionada a la situación, se reduce la probabilidad de lograr un adecuado afrontamiento. Esto puede deberse a la interferencia para controlar los pensamientos automáticos irracionales (p.ej. "no voy a poder” o "no valgo”) y otros síntomas vegetativos asociados a la respuesta ansiosa (p.ej. temblor, sudores o tartamudeo) (ver Spielberger, 1979). Diversos estudios han demostrado que cuando el nivel de ansiedad es elevado, el

(cc) EY-NC-ND 2018, Universitat Politècnica de València

Congreso IN-RED (2018) 
rendimiento académico disminuye, especialmente en situaciones valoradas por el estudiante como difíciles o potencialmente ansiosas (Eysenck, 2013; Sommers-Flanagan y SommersFlanagan, 2012; Sue, 1996).

Otra variable altamente asociada al rendimiento académico, y que predice el éxito posterior es la autoeficacia (Bandura, 1997), que se define como la capacidad percibida para hacer frente a situaciones o tareas concretas. Los alumnos que presentan una baja autoeficacia son más propensos a obtener peores resultados (Bandura, 1992). A su vez, la percepción de incapacidad para alcanzar las metas académicas incrementa la probabilidad de experimentar ansiedad. Por ello autoeficacia y ansiedad están estrechamente relacionadas, y ambas influyen en el rendimiento académico.

Desde el ámbito educativo, diversos estudios han explorado el papel de la ansiedad y la autoeficacia para una mejor comprensión de los mecanismos que facilitan o dificultan la ejecución del alumno (Álvarez y Muñoz, 2018; Contreras et al., 2005; Sue, 1996). A menudo la ansiedad y la autoeficacia suelen estudiarse desde el punto de vista del alumno con el fin de mejorar las prácticas pedagógicas y de aprendizaje. Sin embargo, a pesar de tratarse de variables subjetivas en su evaluación y con un gran peso en el rendimiento académico, no han sido exploradas desde una perspectiva multi-informante en una actividad propuesta desde un curso e-learning. Además de la auto-valoración del propio alumno, sería interesante obtener la valoración de los compañeros del curso y del profesor sobre el desempeño de éste, su nivel de ansiedad y autoeficacia para aplicar habilidades y obtener éxito en la tarea. La aplicación de una estrategia multi-informante es altamente recomendable para realizar una evaluación más exhaustiva y completa (Gómez, 2004). Además, permite comparar las evaluaciones de los informantes y reflexionar sobre posibles diferencias observadas entre lo que uno percibe de sí mismo y lo que los demás observan en su desempeño.

\section{Objetivos}

El objetivo principal de este estudio fue describir el proceso de evaluación desde una perspectiva multi-informante de la ansiedad, autoeficacia y desempeño global de alumnos de postgrado para realizar una actividad práctica (propuesta a través de la plataforma Edx). Los objetivos específicos fueron: (a) analizar posibles diferencias en las evaluaciones de ansiedad, autoeficacia y desempeño global de la tarea entre los tres informantes (el propio alumno, sus compañeros y el profesor), y (b) analizar la relación entre la edad del alumno y su ansiedad, autoeficacia y desempeño para realizar la tarea (y analizar posibles diferencias entre los informantes).

A partir de estudios previos que indican una baja consistencia entre las evaluaciones realizadas por diferentes informantes (Gómez, 2004) y lo expuesto anteriormente se hipotetizó que: a) Habrá diferencias en la valoración de ansiedad, autoeficacia y desempeño global entre los tres evaluadores. Sería esperable que, en la autoevaluación, los alumnos indicarán un mayor nivel de ansiedad, menor autoeficacia y desempeño respecto a las valoraciones de los compañeros y los profesores; y b) A mayor edad (entendida aquí como mayor experiencia, aunque no necesarimente) menor ansiedad, mayor autoeficacia y mejor desempeño en la tarea. 


\section{Desarrollo de la innovación}

\subsection{Procedimiento}

En el curso académico vigente arrancó por primera vez la modalidad online del Máster en Terapia Psicológica con Niños y Adolescentes (http://mtpna.edu.umh.es/). Puesto que esta modalidad debe ser equivalente a la presencial, avalada por la Agencia Nacional de Evaluación de la Calidad y Acreditación (ANECA), el equipo docente se ha esforzado por adaptar los contenidos y metodologías docentes aplicados habitualmente en las clases presenciales al entorno virtual.

Una de las principales dificultades encontradas fue la adaptación de los sistemas de evaluación de las actividades prácticas dirigidas al entrenamiento en habilidades y competencias del terapeuta infantil. En este contexto surge una propuesta de innovación docente que consiste en plantear un sistema de evaluación multi-informante en red para proporcionar feedback sobre las habilidades terapéuticas de los alumnos del curso online. En este proceso se implicó a cada alumno en su evaluación (autoevaluación), a los compañeros matriculados y a los profesores de la asignatura con el fin de evaluar la actividad y favorecer el aprendizaje colaborativo.

Con el propósito de mejorar las competencias y habilidades terapéuticas de los alumnos, se planteó una actividad de entrenamiento que culminaba con la elaboración de un vídeo docente. Cada alumno debía grabarse en una situación simulada (previamente descrita y trabajada con el profesor) en la que actuaba como terapeuta junto a un voluntario (que hacía de paciente). Se mostraba cómo el terapeuta interaccionaba con el paciente para resolver una casuística en particular. En el plazo establecido, cada alumno enviaba el vídeo al equipo docente siguiendo las instrucciones. Además, el alumno evaluaba online su ejecución a partir del visionado de su vídeo. Todas las grabaciones fueron guardadas en una carpeta de Drive compartida, a la que los alumnos podían acceder para visualizar su vídeo y el del resto de compañeros. Los vídeos presentaban situaciones comunes que pueden darse en el contexto de una consulta psicológica y que los profesores valoraron su manejo como interesante para su formación. Posteriormente, cada alumno evaluaba a través de un formulario online a dos compañeros (uno que tenía la misma situación terapéutica y otro con una situación diferente). Esto les permitía, además de tener resueltos diferentes casos clínicos, aprender nuevas formas de afrontar una misma situación. Paralelamente, el profesor evaluaba los vídeos para puntuar la actividad. Todas las evaluaciones se realizaron online a través de Google Forms. En la Figura 1 se muestra gráficamente el proceso:

(cc) EY-NC-ND 2018, Universitat Politècnica de València

Congreso IN-RED (2018) 
1

- Actividad de entrenamiento en habilidades terapéuticas para resolver casos clínicos

- Alumnos envían su vídeo docente y el formulario de autoevaluación online

3 - Asignación de los compañeros que debe evaluar cada alumno por parte del profesor

4 - Evaluación de las competencias de los alumnos por parte de sus compañeros

5 • Evaluación de las competencias de los alumnos por parte del profesor

6

- Envío de las evaluaciones de los compañeros y del profesor a cada alumno

7

- Evaluación final de la actividad docente

Fig 1. Proceso de evaluación de las competencias

\subsection{Participantes}

Todos los alumnos de la modalidad online $(N=30)$ fueron invitados a participar en esta actividad de innovación docente. Salvo dos hombres, el resto eran mujeres. La edad del grupo osciló entre los 22 y 46 años $(M=29.79 ; D T=7.17)$. Hubo dos alumnas internacionales (Argentina y Ecuador) y los demás residían en España distribuidos en diferentes áreas geográficas.

\subsection{Medidas de evaluación}

Se realizaron tres evaluaciones online simultáneas de cada vídeo docente: autoevaluación (cada alumno sobre su ejecución), la evaluación de los compañeros y la evaluación del profesor. Todos respondieron a las mismas preguntas pero en formularios independientes y haciendo referencia siempre al alumno de cada vídeo. El evaluador valoraba lo siguiente:

- Ansiedad percibida en el terapeuta. La respuesta comprendía un rango de 0 (nada ansioso/a) a 10 (muy ansioso/a).

- Capacidad del terapeuta para aplicar las habilidades entrenadas (autoeficacia). La respuesta comprendía un rango de 0 (muy mala) a 10 (muy buena).

- Capacidad del terapeuta para resolver con éxito el caso clínico (autoeficacia). La respuesta comprendía un rango de 0 (muy mala) a 10 (muy buena).

- Valoración global de la actuación del terapeuta (desempeño). La respuesta comprendía un rango de 0 (muy mala) a 10 (muy buena).

- Edad del alumno autoinformada en años. 


\subsection{Análisis de datos}

Se realizaron análisis sencillos para obtener la media en las evaluaciones de los propios alumnos, de los compañeros y del profesor en las variables: ansiedad percibida, autoeficacia (para aplicar las habilidades y para resolver con éxito el caso clínico) y la evaluación gobal. Los análisis de correlación bivariada de Spearman (rho) permitieron evaluar si existía relación entre la edad de los alumnos, y el desempeño del alumno en la actividad desde el punto de vista del alumno, de los compañeros y del profesor. Todos los análisis se realizaron con el paquete estadístico SPSS v25.

\section{Resultados}

En la Tabla 1 se muestran las medias y desviaciones típicas para cada variable evaluada en el estudio desde una perspectiva multi-informante: alumno, compañeros y profesor. En lo que respecta al nivel de ansiedad del terapeuta, se observa una diferencia estadísticamente significativa entre los tres informantes. Los alumnos se percibieron más nerviosos en la grabación (autoevaluación) respecto a la evaluación de los compañeros y del profesor. En la valoración de la autoeficacia observamos que los alumnos tendieron a puntuarse ligeramente por debajo de la puntuación emitida por el profesor y sus compañeros. Esto sugiere que el profesor y los compañeros perciben a los alumnos más capaces de aplicar las habilidades terapéuticas y resolver con éxito el caso clínico que los propios alumnos (evaluándose a ellos mismos). Resultados similares fueron encontrados en la variable de valoración global de la actuación del terapeuta. El profesor puntuó más positivamente la actuación del terapeuta que los alumnos su propia ejecución.

Tabla 1. Evaluación multifuente del entrenamiento en resolución de casos clínicos

\begin{tabular}{|c|c|c|c|c|}
\hline Evaluador & $\begin{array}{c}\text { Ansiedad } \\
\text { percibida } \\
M(D T)\end{array}$ & $\begin{array}{c}\text { Autoeficaci } \\
\text { a percibida } \\
\text { para } \\
\text { aplicar las } \\
\text { habilidades } \\
M(D T)\end{array}$ & $\begin{array}{c}\text { Autoeficaci } \\
\text { a percibida } \\
\text { para } \\
\text { resolver } \\
\text { con éxito el } \\
\text { caso } \\
M(D T)\end{array}$ & $\begin{array}{c}\text { Valoración } \\
\text { global } \\
M(D T)\end{array}$ \\
\hline Alumno (autoevaluación) & $5.26(2.45)$ & $7.41(1.42)$ & $7.74(.90)$ & $7.37(1.41)$ \\
\hline Compañeros de clase & $2.92(2.11)$ & $7.92(1.09)$ & $8.07(1.03)$ & $8.04(.99)$ \\
\hline Profesores de la asignatura & $2.29(1.36)$ & $8.11(1.19)$ & $8(1.26)$ & $8.17(1.13)$ \\
\hline
\end{tabular}

En la Tabla 2 se observan los coeficientes de correlación de Spearman entre la edad de los alumnos, y la ansiedad percibida, la autoeficacia y la valoración general. Solo hubo correlaciones indirectas y estadísticamente significativas entre la edad de los alumnos y la autoeficacia percibida para resolver con éxito la evaluación y la valoración global en el caso de la autoevaluación de los alumnos. Esto indica que cuando los alumnos evaluaron su propia ejecución como terapeutas tendieron a evaluarse más negativamente y a percibirse menos capaces de resolver con éxito el caso clínico a medida que tienen más edad. En otras

(cc) EY-NC-ND 2018, Universitat Politècnica de València

Congreso IN-RED (2018) 
palabras, los alumnos de mayor edad tendieron a autoevaluarse más negativamente que los compañeros de menor edad. Sin embargo, es destacable que no existe asociación entre la edad y las variables analizadas en las evaluaciones de los compañeros y el profesor. Por tanto, la edad no estuvo relacionada con la evaluación emitida sobre la ansiedad, la autoeficacia y el desempeño por parte de los compañeros y el profesor.

Tabla 2. Relación entre el desempeño del alumno y la edad del alumno para cada informante: alumnos, compañeros y el profesor

\begin{tabular}{lcccc}
\hline Evaluador & Ansiedad & $\begin{array}{c}\text { Autoeficacia } \\
\text { percibida } \\
\text { para aplicar } \\
\text { las }\end{array}$ & $\begin{array}{c}\text { Autoeficacia } \\
\text { percibida } \\
\text { para } \\
\text { resolver con } \\
\text { habilidades }\end{array}$ & $\begin{array}{c}\text { Valoración } \\
\text { global }\end{array}$ \\
\hline Alumno (autoevaluación & & \\
Compañeros de clase & .28 & -.08 & $-.43^{*}$ & $-.41^{*}$ \\
Profesores de la asignatura & .17 & -.09 & .01 & -.25 \\
\hline$p<.05$. & -.06 & .03 & -.03 & -.09 \\
\hline
\end{tabular}

El presente trabajo describe el proceso de evaluación multi-informante aplicado al entrenamiento de las competencias de los terapeutas infantiles en un curso e-learning. Los resultados de esta experiencia piloto confirman la hipótesis inicial, que indica que la evaluación de la ansiedad, la autoeficacia y el desempeño difiere en función del informante: los propios alumnos (autoevaluación), la valoración de los compañeros y la del profesor. Los datos sugieren que los alumnos tienden a evaluar sus habilidades y competencias con prudencia y honestidad, si tomamos en cuenta que los otros informantes presentaron independientemente calificaciones similares. Por lo tanto, podrían considerarse las respuestas de autoevaluación bastante objetivas.

Conviene destacar que los alumnos se perciben significativamente más ansiosos en la grabación respecto a la evaluación de los compañeros y el profesor, que los perciben significativamente menos ansiosos. Una explicación a este resultado podría ser que los alumnos estén evaluando el nivel de ansiedad que recuerdan haber experimentado en la situación de grabación, y no realmente la ansiedad que perciben en ellos mismos en el vídeo. Los signos de ansiedad visibles (p.ej. sudoración excesiva, tartamudeo o automanipulaciones) pueden estar menos presentes en personas que tienen un adecuado manejo de la ansiedad, como podría ser el caso de alumnos de psicología, que a menudo se exponen a hablar en público. Por otro lado, la presencia de signos de ansiedad no visibles (p.ej. palpitaciones o pensamientos negativos intrusivos) pudo explicar las diferencias observadas entre los informantes. La ansiedad se considera una respuesta adaptativa para afrontar las demandas del ambiente; no obstante, cuando el nivel de ansiedad no se corresponde con la amenaza real, ésta tiene efectos negativos en la capacidad del individuo 
para resolver situaciones (ver Sommers-Flanagan y Sommers-Flanagan, 2012). En este sentido, las autovaloraciones de los alumnos sobre su capacidad para aplicar las habilidades entrenadas y para resolver el caso clínico son positivas, si bien, algo inferiores (si tomamos la media) con respecto a la valoración de los compañeros y los profesores. En consecuencia, percibirse ansioso para resolver el caso clínico pudo afectar a la percepción de la capacidad para resolverlo correctamente (autoeficacia) en los alumnos. En lo que respecta a la valoración global, las evaluaciones de todos los informantes fueron similares y positivas (calificación media de notable), aunque la del profesor fue ligeramente más positiva que la de los alumnos (autoevaluación) y los compañeros.

En este estudio se muestra que la edad del alumno influye en su propia percepción de autoeficacia para resolver con éxito la situación y su desempeño global como terapeuta infantil. Concretamente los alumnos de mayor edad tendieron a evaluarse más negativamente y a percibirse menos capaces de resolver con éxito el caso clínico. Este resultado no confirma nuestra hipótesis inicial. Sin embargo, esta asociación entre edad y competencia del terapeuta no se refleja en las evaluaciones de los compañeros y la del profesor. Estas diferencias en la autoevaluación de los alumnos pueden deberse a que los más mayores son más exigentes con la evaluación de su competencia como terapeutas. No obstante, también sería esperable un mejor manejo de la situación si se sobreentiende una relación entre la experiencia práctica y la edad (aunque no siempre es así). Mayor evidencia empírica es necesaria para extraer resultados concluyentes.

\section{Conclusiones}

A partir de esta propuesta de innovación docente se pueden extraer al menos tres conclusiones de los resultados: 1) La evaluación de una actividad difiere según el evaluador (el propio alumno, los compañeros o el profesor), 2) Ante una misma situación, los propios alumnos tienden a percibirse más ansiosos, menos autoeficaces y con un peor desempeño respecto a las evaluaciones de los compañeros y del profesor, y 3) Los alumnos con mayor edad se percibieron menos autoeficaces para resolver la situación con éxito y valoraron más negativamente su desempeño global que los más jóvenes. Sin embargo, las evaluaciones de los compañeros y del profesor no estuvieron relacionadas con la edad del alumno.

En base a lo mencionado anteriormente, el proceso de evaluación multi-informante se considera interesante para el aprendizaje por varias razones. Nos permite obtener información de utilidad sobre las percepciones y creencias de los alumnos asociadas a su proceso de aprendizaje y autoeficacia. Paralelamente se establece una vía de aprendizaje colaborativo, en la que los alumnos proporcionan feedback unos a otros, y aprenden de la experiencia del otro y observando cómo lo hacen otros. Como ya indicaba Bandura (1986), el modelado (imitación o aprendizaje observacional) es una de las principales formas de aprendizaje. Teniendo en cuenta que los cursos e-learning presentan riesgo de convertirse en procesos de aprendizaje solitarios (en comparación con las clases presenciales), tener la oportunidad de aprender de otros e interaccionar es fundamental. En esta línea, los vídeos docentes grabados por nuestros alumnos están en proceso de edición para ser parte del material docente el próximo año. 
Esta experiencia docente puede extrapolarse a cualquier nivel educativo, desde primaria a postgrado, adaptando el proceso de evaluación a las características del alumnado y de la actividad propuesta. Puede ser especialmente recomendable para la evaluación de habilidades prácticas de atención al paciente (p.ej. Grado de Medicina, Grado de Enfermería y Grado en Terapia Ocupacional) y para el entrenamiento de habilidades de comunicación en general. Desde una perspectiva psicológica, la metodología de esta actividad es positiva para que los alumnos relativicen el nivel de ansiedad percibida y su autoeficacia para realizar con éxito una tarea en el contexto académico. A menudo, los propios alumnos son más críticos con su propia ejecución que otros observadores externos. Por otro lado, recibir feedback positivo y críticas constructivas por parte de los compañeros, además del profesor, contribuye al aprendizaje colaborativo, así como a fortalecer la autoestima y la autoeficacia de los propios alumnos.

En futuros proyectos está previsto favorecer la interacción de los alumnos de este curso elearning en sesiones presenciales con los alumnos de la modalidad presencial y a través de nuevas aplicaciones móviles como MyLearningMentor (Alario-Hoyos, Estévez-Ayres, Pérez-Sanagustín, Leony y Kloos, 2015) y MyMOOCSpace (Ramírez-Donoso et al., 2015; Ramírez-Donoso et al., 2017). Nuevas formas de evaluación de los aprendizajes prácticos a través de los entornos virtuales deben explorarse para su incorporación próximamente.

\section{Agradecimientos}

El Programa de Innovación Educativa Universitaria (PIEU-UMH 2017-18) de la Universidad Miguel Hernández, España (Ref. PIEU2017/24), ha apoyado económicamente este trabajo.

\section{Referencias}

ALARIO-HOYOS, C., ESTÉVEZ-AYRES, I., PÉREZ-SANAGUSTÍN, M., LEONY, D. y KLOOS, C. D. (2015). "MyLearningMentor: A Mobile App to Support Learners Participating in MOOCs”. Journal of Universal Computer Science, vol. 21, issue 5, p. 735753.

ÁLVAREZ, I. M. y MUÑOZ, A. L. (2018). Estudio neuropsicológico de la funcionalidad visual, las estrategias de aprendizaje y la ansiedad en el rendimiento académico. Aula abierta, vol. 47, issue 2, p. 245-254.

BANDURA, A. (1982). Self-efficacy mechanism in human agency. American Psychologist, vol. 37, issue 2, p. 122-147.

BANDURA, A. (1996). Teoría del aprendizaje social. Madrid: Espasa-Calpe, S.A

BANDURA, A. (1997). Self-efficacy: The exercise of control. New York: N.H. Freeman.

CONTRERAS, F., ESPINOSA, J. C., ESGUERRA, G., HAIKAL, A., POLANÍA, A. y RODRÍGUEZ, A. (2005). Autoeficacia, ansiedad y rendimiento académico en adolescentes. Diversitas: Perspectivas en Psicología, vol.1, issue 2, p. 183-194.

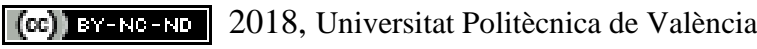


"Cuanto más practiques, mejor lo harás":

evaluación multi-informante en el contexto de un curso e-learning

EYSENCK, M. W. (2013). Anxiety: The cognitive perspective. Hove, UK: Psychology Press.

GÓMEZ, I. (2004). Evaluación Infantil Cognitivo-Comportamental. Evaluación Multimetodal y Multinforme. Revista Neuropsicología, Neuropsiquiatría y Neurociencias, vol. 6, issue 1, p. 21-25.

HERNÁNDEZ, N. y CARRIL, P. C. (2012). Trabajo colaborativo en entornos e-learning y desarrollo de competencias transversales de trabajo en equipo: Análisis del caso del Máster en gestión de Proyectos en Cooperación Internacional, CSEU La Salle. $<$ http://openaccess.uoc.edu/webapps/o2/handle/10609/26321> [Consulta: 27 de marzo de 2018]

ORGILÉS, M. (2014). Tratando... fobia a la oscuridad en la infancia y adolescencia. Madrid: Ediciones Pirámide.

RAMÍREZ-DONOSO, L., PÉREZ-SANAGUSTÍN, M., NEYEM, A. y ROJAS-RIETHMULLER, J. S. (2015, octubre). "Promoviendo la colaboración efectiva en MOOCs a través de aplicaciones móviles” en Congreso IEEE Chilecon 2015, Santiago de Chile, Chile). Disponible en $<$ https://www.researchgate.net/publication/280778018_Promoviendo_la_Colaboracion_Efe ctiva_en_MOOCs_a_traves_de_Aplicaciones_Moviles> [Consulta: 27 de marzo de 2018]

RAMÍREZ-DONOSO, L., ROJAS-RIETHMULLER, J. S., PÉREZ-SANAGUSTÍN, M., NEYEM, A. y ALARIO-HOYOS, C. (2017). "MyMOOCSpace: A cloud-based mobile system to support effective collaboration in higher education online courses”. Computer Applications in Engineering Education, vol. 25, issue 6, p. 910-926.

SOMMERS-FLANAGAN, J. y SOMMERS-FLANAGAN, R. (2012). Counseling and psychotherapy theories in context and practice: Skills, strategies, and techniques ( ${ }^{\text {nd }}$ ed.). New Jersey: John Wiley \& Sons

SPIELBERGER, C. (1979). Tensión y ansiedad. México: Harla, S.A.

SUE, D. (1996). Comportamiento anormal. Mexico: Mc Graw-Hill.

UAB (Universidad Autónoma de Barcelona) (2017). MOOC. Diferencias con los cursos online. $<\underline{\text { http://www.uab.cat/web/estudiar/mooc/diferencias-con-un-curso-online- }}$ $\underline{1345668281279 . h t m l}>$ [Consulta: 25 de marzo de 2018]

(cc) EY-NC-ND 2018, Universitat Politècnica de València

Congreso IN-RED (2018) 